\title{
Overgeneral autobiographical memory predicts diagnostic status in depression
}

\author{
Dirk Hermans $^{\mathrm{a}, *}$, Heleen Vandromme ${ }^{\mathrm{a}}$, Elise Debeer ${ }^{\mathrm{a}}$, Filip Raes ${ }^{\mathrm{a}}$, \\ Koen Demyttenaere ${ }^{\mathrm{b}}$, Els Brunfaut ${ }^{\mathrm{b}}$, J. Mark G. Williams ${ }^{\mathrm{c}}$ \\ ${ }^{a}$ Department of Psychology, University of Leuven, Tiensestraat 102, 3000 Leuven, Belgium \\ ${ }^{\mathrm{b}}$ University Psychiatric Centre, Leuven, Belgium \\ ${ }^{\mathrm{c}}$ University of Oxford, $U K$
}

Received 26 November 2007; received in revised form 21 January 2008; accepted 29 January 2008

\begin{abstract}
Major depressive disorder (MDD) is characterised by difficulties in retrieving specific autobiographical memories, with a significant propensity towards categoric memories (i.e. memories of a summary type). Previous studies have demonstrated that this overgeneral memory is a valid predictor of the course of depression, with reduced specificity being associated with worse outcome. Most of these studies have employed continuous measures of depression to assess the course of the symptoms. This study investigated whether overgeneral memory also predicts clinical status at follow-up (i.e. whether patients still meet criteria for depression). Patients who fulfilled criteria for major depressive disorder were tested shortly after admission to the hospital and were retested some weeks later. It was found that lower levels of specificity or a higher number of categoric memories were associated with a higher probability of still being diagnosed with MDD. These memory variables outperformed other relevant indices, such as depression severity, rumination, level of self-esteem and dysfunctional attitudes.

(C) 2008 Elsevier Ltd. All rights reserved.
\end{abstract}

Keywords: Autobiographical memory; Memory specificity; Depression; Rumination

\section{Introduction}

There exists a considerable body of evidence that shows that clinical depression is characterised by overgeneral autobiographical memory. The cue word methodology that is typically used in these studies is known as the Autobiographical Memory Test (AMT) (Williams \& Broadbent, 1986). It consists of a series of cue words, alternating in valence (e.g. enjoy, lazy, surprised), for which participants are asked to come up with a specific memory. They are told that the event recalled should be a specific event, something that happened at a particular place and time and lasted for a day or less. In spite of clear instructions, persons suffering from major depressive disorder (MDD) respond with more memories of a summary type (i.e. categoric memories)

\footnotetext{
*Corresponding author. Tel.: + 3216325963 ; fax: + 3216326099.

E-mail address: dirk.hermans@psy.kuleuven.be (D. Hermans).
} 
as compared with controls. An example of a specific memory for the cue word 'lonely' is 'I really felt lonely last Friday when nobody turned up at the party', while a categoric memory for the same cue word would be 'I feel lonely on the evenings that my wife goes out working'. A recent overview of the available studies revealed that this difference between depressed patients and matched controls yields a large effect size (Cohen's $d=1.12$ ) (Williams et al., 2007).

The observation that depression is characterised by overgeneral autobiographical memory has received a lot of attention. One of the reasons is that overgeneral memory has been found to predict clinical outcome in depression. Brittlebank, Scott, Williams, and Ferrier (1993) were the first to report that patients with MDD who retrieved more overgeneral memories on the AMT had a poorer prognosis. Overgeneral memory at baseline significantly predicted poorer long-term outcome at 3 and 7 months of follow-up. This effect was present even when initial symptom levels were taken into account. Other studies have replicated the results of Brittlebank et al. (but see Brewin, Reynolds, \& Tata, 1999, for a non-replication). In a group of depressed outpatients, Peeters, Wessel, Merckelbach, and Boon-Vermeeren (2002) showed that a lower number of specific responses (on negative cues) was predictive of worse outcome at 3 and 7 months of follow-up, over and above initial symptom levels. Similarly, Raes et al. (2006) showed that reduced specificity to negative cue words predicted higher levels of depression at 7 months of follow-up, even when baseline depression scores were partialled out. Dalgleish, Spinks, Yiend, and Kuyken (2001) extended these findings to a group of patients who suffered from seasonal affective disorder. Finally, Raes et al. (in press) observed that - after controlling for relevant variables - categoric memories predicted a less favourable course after electroconvulsive therapy in a group of depressed inpatients.

Interestingly, similar results have been reported outside the domain of clinical depressive disorder. For a variety of populations it has been observed that autobiographical memory performance significantly predicts changes in emotional responding over the course of weeks or months (e.g. Harvey, Bryant, \& Dang, 1998; Hipwell, Reynolds, \& Pitts Crick, 2004; Mackinger \& Svaldi, 2004). Based on this extensive series of studies, one can conclude that the level of autobiographical memory specificity is an important variable in predicting the course of emotional responses in clinical and non-clinical groups. Nevertheless, it has to be noted that the number of studies that directly target the relation between autobiographical memory and the course of clinical depression is still relatively limited. Given the theoretical and clinical relevance of elucidating the mechanisms of change in depression, additional work in this area is essential. This is particularly so given that some issues remain open for further empirical investigation. One question relates to the type of dependent variable that is used to assess the outcome of depression. The second concerns the relationship between autobiographical memory and rumination.

With respect to the dependent variables, it is important to note that in all studies that investigated the predictive power of autobiographical memory in currently depressed patients, symptom measures of depression were employed to assess changes over time. Examples are the Beck Depression Inventory (BDI; Beck, Steer, \& Brown, 1996) and the Hamilton Rating Scale for Depression (HRSD; Hamilton, 1960). Although it is an evident choice to assess changes in depression using continuous symptom measures like the BDI or the HRSD, the fact that no previous studies employed a more categorical (i.e. diagnostic) measure is at least surprising. In fact, the overall pattern that emerges from studies that investigated the relation between depression and autobiographical memory is that the diagnostic status of the patients (i.e. presence or absence of a formal diagnosis of clinical depression) is associated with overgeneral memory, rather than the level of depression (Williams et al., 2007). From this perspective it remains untested whether overgeneral memory also predicts the course of depression, when this is defined in terms of whether patients still fulfil diagnostic criteria for clinical depression. Moreover, a recent discussion about what exact features of depression are predicted by the AMT has revealed that the choice of the dependent variable might be of central concern (see Discussion for more details).

A second issue that emanated from the study of the relation between overgeneral memory and the course of depression pertains to the role of rumination. Several studies have indicated that this cognitive behaviour pattern, which is one of the central characteristics of depression, is closely related to overgeneral memory. In addition to correlational studies (e.g., Ramponi, Barnard, \& Nimmo-Smith, 2004), experimental work has corroborated this relationship (e.g. Watkins \& Teasdale, 2001). Given this intimate relation, Raes et al. (2006) included the Rumination on Sadness Scale (RSS) (Conway, Csank, Holm, \& Blake, 2000) as a measure of 
ruminative processing in their prediction study. When the effect of rumination was partialled, memory specificity no longer predicted level of depression at follow-up. Based on additional analyses, Raes et al. concluded that this is a first indication of rumination as one possible pathway by which reduced AM specificity exerts its negative effects on the course of depression. As a matter of fact, in the Capture and Rumination, Functional Avoidance and Executive Control (CaRFAX) model that was recently put forward, vulnerability to capture errors leading to rumination was identified as one of three mechanisms that underlie the effects of overgeneral memory on depression (Williams et al., 2007).

In light of the above, our present study was undertaken with a threefold focus. First, we investigated whether additional evidence could be provided for the observation that overgeneral memory predicts the course of depression. Second, we examined whether this effect would extend to the prediction of the diagnostic status of the patients at follow-up, rather than the level of depressive symptoms. Third, we assessed whether these effects occur independently of rumination. To test these hypotheses, a group of inpatients who fulfilled criteria for MDD was tested after admission to the hospital and some weeks later. We investigated whether AMT at Time 1 predicted diagnostic status at Time 2. In addition to other variables (e.g. self-esteem, dysfunctional attitudes, depression severity, previous depressive episodes), ${ }^{1}$ the independent effect of rumination was assessed by means of the RSS.

\section{Method}

\section{Participants}

Twenty-six patients (14 women) participated in this study. The mean age of the sample was 39.9 years $(\mathrm{SD}=10.8$; range $=24-59)$. They were inpatients at the University Psychiatric Centre (University of Leuven) and started treatment for depression. Inclusion criteria were a DSM-IV diagnosis of MDD (APA, 1994) and an age between 18 and 65 years. Patients were interviewed using the mood modules of the Structured Clinical Interview for DSM-IV (SCID; First, Gibbon, Spitzer, \& Williams, 1996; Dutch version: van Groenestijn, Akkerhuis, Kupka, Schneider, \& Nolen, 1999). This interview was conducted by two trained students (Master's in Clinical Psychology). At first assessment, all but three patients were receiving antidepressant medication. For 15 of the 26 participants there were indications of comorbidity (e.g. GAD, sleep apnoea, diabetes mellitus, social phobia, alcohol abuse).

\section{Materials}

The Autobiographical Memory Test (AMT)

An extended version of the Dutch AMT was used (Raes et al., 2006; original version: Williams and Broadbent, 1986). Participants were asked to recall a specific memory to 18 cue words. Words were presented orally in a fixed order, with nine positive and nine negative words alternating: happy, sad, safe, angry, interested, clumsy, successful, emotionally hurt, surprised, lonely, relaxed, guilty, proud, scared, pleasurable, cowardly, carefree and lazy. ${ }^{2}$ Positive and negative cues were matched for familiarity, imageability and emotional extremity. The instructions state that a specific memory refers to one particular personally experienced event that happened on a particular day at least 1 week before. Participants were instructed not to retrieve the same memory twice. They were given $60 \mathrm{~s}$ for each cue. If the first response was not a specific memory, participants were verbally prompted to describe a particular time or a particular event. The prompting procedure was repeated until the participant retrieved a specific memory or until the time limit was exceeded. To familiarize participants with the procedure, three practice words were given (enjoy, friendly and naughty).

\footnotetext{
${ }^{1}$ In addition to self-esteem (SLCS-R), dysfunctional attitudes (DAS), depression severity (BDI-II) and rumination (RSS), it was also assessed whether patients had experienced previous episodes of depression (yes $=17 /$ no $=9$ ) and whether they had been hospitalised for previous episodes (yes $=8 /$ no $=18$ ). Both variables were first included in the crucial logistic regressions. However, because there was no significant contribution for these variables, and because the results were identical whether or not these variables were taken into account, it was decided not to include them in the analyses that will be reported.

${ }^{2}$ The Dutch words can be obtained from the first author.
} 
Each response was later coded as a specific or non-specific memory. The latter were further qualified as a categoric memory, an extended memory (e.g., 'The three years that I worked in the coalmine'), no memory (e.g., a verbal association such as 'my father'), omission, same event (referring to an event already mentioned) or incorrect specific (referring to an event of the past week). Using this scoring procedure, we obtained good reliability ( $K=0.96$; Raes, Hermans, de Decker, Eelen, \& Williams, 2003).

\section{Beck Depression Inventory (BDI-II)}

The BDI-II (Beck et al., 1996) is a widely used self-rating measure to assess the severity of depressive symptoms and consists of 21 four-choice statements (range $=0-63$ ). The Dutch version by Van der Does (2002) is used, which has adequate reliability (Cronbach's $\alpha$ of 0.92 and 0.93 in a psychiatric and student population, respectively).

\section{Dysfunctional Attitude Scale (DAS)}

The original DAS (Weissman \& Beck, 1978) is a 100-item self-report measure developed to assess dysfunctional cognitions related to depression. In the present study, a Dutch translation of the DAS-A was employed (Raes, Hermans, Van den Broeck, \& Eelen, 2005). Cronbach's $\alpha$ of this version in a group of patients suffering from MDD was 0.94 .

\section{Rumination on Sadness Scale ( RSS)}

The RSS (Conway et al., 2000) is a 13-item questionnaire measuring the tendency to react to sad mood by ruminating. The RSS has demonstrated good internal consistency and good convergent and discriminant validity (for more details, see Conway et al., 2000). The Dutch translation by Raes, Hermans, and Eelen (2003) was used $(\alpha=0.84)$. Both the original RSS $(r=0.81)$ and the Dutch version $(r=0.77)$ correlate significantly with the Ruminative Response Scale (Nolen-Hoeksema \& Morrow, 1991).

\section{Self-Liking/Self-Competence Scale Revised (SLCS-R)}

The SLSC-R is a self-report questionnaire (Tafarodi \& Swann, 2001), which contains eight items for each of the two dimensions of self-esteem, namely Self-Competence and Self-Liking. We used the Dutch version of the SLCS-R, which has appropriate reliability and validity (Vandromme, Hermans, Spruyt, \& Eelen, 2007).

\section{Procedure}

Participants were tested twice. The pre-test was scheduled as soon as possible after admission to the hospital, usually during the first or second week. All participants gave informed consent and were tested individually. The mood section of the SCID was administered first, after which the AMT was completed, followed by the BDI-II. The DAS, RSS and SLCS-R were given to the participants, who were allowed to complete these questionnaires in their room, after which they were returned to the experimenter. The post-test was conducted 3-4 weeks after the first test session. The measures used during this second session were identical to those of the pre-test. The mean number of days between the sessions was 24.3 days $(\mathrm{SD}=7.1)$.

Between pre- and post-test, 14 of the 26 participants followed a cognitive-behavioural group treatment for depression in addition to the standard treatment programme (which includes elements like family therapy, psychomotor therapy and general behavioural activation, in addition to medication or individual psychotherapy sessions if indicated). This consisted of five 1.5-h group sessions that focused on cognitive and behavioural aspects of depression. Because only about half of the patients in this study participated in this group treatment, this was included as a variable in all analyses. For the participants who followed the group sessions, the pre- and post-tests were scheduled immediately before and after this group treatment. For the remaining participants, a similar time interval was used. Both subgroups did not differ for the length of the interval between both sessions, $t(24)=1.87$, n.s. The timing of the test sessions was unrelated to symptom severity, as was also the case for the assignment to one of the two groups. 


\section{Results}

\section{Data analysis}

The crucial dependent variable in this study was whether patients still fulfilled DSM criteria for MDD at Time 2. Given that this constitutes a binary variable $(1=$ yes; $0=$ no), logistic regression analyses were performed to evaluate the contribution of the predictors.

\section{Participant characteristics}

Table 1 presents all means for the questionnaire data. At pre-test, all participants were diagnosed as suffering from MDD. The mean score of the BDI-II $(M=29.7)$ indicates that this group is characterised by significant levels of depressive symptoms. According to Beck et al. (1996), BDI-II scores above 28 should be regarded as indicative of 'severe depression'. During the course of the inpatient treatment programme, the BDI scores dropped significantly to a mean score of 18.8, which indicates a 'mild' level of depression. A similar significant improvement was observed for the rumination scores. For the measures of dysfunctional attitudes (DAS) and self-esteem (SLCS-R), improvements were observed, which did not, however, reach the level of statistical significance.

When patients were tested for the second time with the SCID (Time 2), 18 of the 26 patients no longer fulfilled criteria for MDD. Follow-up tests indicated that both groups (i.e. those who did and those who did not fulfil criteria at Time 2) did not differ on any of the questionnaire measures at Time 1; all $t$ 's $<1.3$, all $p$ 's $>0.27$. We can thus conclude that, compared with those who did not recover from major depression, those who did recover were not characterised by higher or lower initial levels of depression, rumination or dysfunctional attitudes, and did not differ in levels of self-esteem.

\section{AMT Performance}

Approximately $70 \%$ of the first responses to AMT cues at baseline were specific (Table 2). About $16 \%$ of the first responses were overgeneral memories (categoric + extended), which is somewhat lower than the

Table 1

Mean scores (and standard deviations) on the BDI-II, DAS, SLCS-R and RSS at Times 1 and 2

\begin{tabular}{|c|c|c|c|}
\hline Variable & Time $1(n=26)$ & Time $2(n=26)$ & Repeated-measures ANOVA \\
\hline BDI-II & $29.69(11.0)$ & $18.84(11.9)$ & $F=21.91, p<0.001$ \\
\hline DAS & $164.50(37.4)$ & $156.15(36.8)$ & $F=2.12, p=0.16$ \\
\hline SLCS-R & $36.04(10.4)$ & 38.04 (11.6) & $F=2.33, p=0.14$ \\
\hline RSS & $47.00(8.30)$ & $42.19(8.02)$ & $F=11.43, p<0.001$ \\
\hline
\end{tabular}

Table 2

Mean number (standard deviations and range) of all response categories for the Autobiographical Memory Test (AMT) at baseline $(n=26)$, including test-retest reliability for all response categories

\begin{tabular}{lrcc}
\hline AMT response category & Mean (SD) & Range & Test-retest \\
\hline Specific & $12.81(4.12)$ & $3-18$ & $0.88^{* *}$ \\
Categoric & $1.88(2.36)$ & $0-7$ & $0.66^{* *}$ \\
Extended & $1.04(1.34)$ & $0-4$ & $0.53^{* *}$ \\
No memory & $0.27(0.45)$ & $0-1$ & $0.48^{*}$ \\
Omission & $1.15(1.62)$ & $0-6$ & $0.82^{* *}$ \\
Same event & $0.54(1.07)$ & $0-5$ & 0.22 \\
Incorrect specific & $0.31(0.62)$ & $0-2$ & 0.23 \\
\hline
\end{tabular}

Note: ${ }^{*} p<0.01,{ }^{*} p<0.05$. 
percentage reported by Raes et al. (2006) using a similar procedure in a different but similar sample (29\%). The mean number of categoric memories was about 2, with a range of $0-7$. The number of specific memories ranged from 3 to $18(M=12.8)$.

Following previous research, correlations were calculated between each of the AMT-response categories and the BDI-II and RSS scores (both at baseline). In line with previous studies (Williams et al., 2007), the level of depression (BDI-II) was unrelated to the number of specific, $r(26)=0.24, p=0.25$, or categoric memories, $r(26)=-0.26, p=0.20$. Also, the level of rumination was not associated with the number of specific, $r(26)=0.22, p=0.29$, or categoric memories, $r(26)=-0.29, p=0.15$.

\section{Autobiographical memory and the prediction of diagnostic status at retest}

Logistic regression analysis was used to test if autobiographical memory predicts whether (1) or not (0) patients were still diagnosed as clinically depressed at Time 2 . We used a hierarchical approach because this allows investigating whether the inclusion of a specific predictor variable leads to a significant improvement of the model.

Because some participants had followed a series of cognitive-behavioural group sessions, whereas others had not, we controlled for the possible influence of this difference by including Group-CBT as a (categorical) predictor in the first block of this hierarchical logistic regression. In the second block of predictors, BDI-II, SLCS-R and DAS were entered. Because of the special status of rumination in this type of research (see Raes et al., 2006), the RSS was not included at this point. We will return to the effects of rumination in the following paragraph. In the final block, the number of categoric memories was entered.

Table 3a presents an overview of the different steps of the regression analysis (block entry approach). In block 1 the Group-CBT variable was entered. Adding this predictor to the constant (block 0) did not significantly improve the model; -2 log-likelihood $(-2 \mathrm{LL})=32.03, \chi^{2}(1)=0.069, p=0.793, R^{2}=0.003$ (Cox and Snell), $R^{2}=0.004$ (Nagelkerke). Likewise, adding the BDI-II, DAS and SLCS-R in block 2 did not lead to a statistically reliable improvement of the model, $-2 \mathrm{LL}=31.26, \chi^{2}(3)=0.769, p=0.857, R^{2}=0.03$ (Cox and Snell), $R^{2}=0.05$ (Nagelkerke). As can be seen from Table $3 \mathrm{a}$, the Wald statistic was not significant for any of the individual predictors entered in this block (BDI-II, DAS, SLCS-R). This indicates that none of these individual predictors contributed to the model. In the third block, the number of categoric memories was

Table 3a

Prediction of diagnostic status by means of categorical memories: regression statistics

\begin{tabular}{|c|c|c|c|c|c|c|}
\hline Predictors & $\beta$ & S.E. $\beta$ & $\operatorname{Exp}(\beta)$ & Wald's $\chi^{2}$ & $\mathrm{Df}$ & $p$ \\
\hline \multicolumn{7}{|l|}{ Block 0} \\
\hline Constant & -0.811 & 0.425 & 0.444 & 3.642 & 1 & 0.056 \\
\hline \multicolumn{7}{|l|}{ Block 1} \\
\hline Constant & -0.223 & 0.851 & 0.500 & 0.069 & 1 & 0.793 \\
\hline Group-CBT & -0.693 & 0.612 & 0.800 & 1.281 & 1 & 0.258 \\
\hline \multicolumn{7}{|l|}{ Block 2} \\
\hline Constant & -0.606 & 3.622 & 0.546 & 0.028 & 1 & 0.867 \\
\hline Group-CBT & -0.375 & 0.907 & 0.687 & 0.171 & 1 & 0.679 \\
\hline BDI & 0.035 & 0.074 & 1.036 & 0.226 & 1 & 0.635 \\
\hline DAS & -0.004 & 0.019 & 0.996 & 0.038 & 1 & 0.846 \\
\hline SLCS-R & -0.013 & 0.056 & 0.546 & 0.054 & 1 & 0.817 \\
\hline \multicolumn{7}{|l|}{ Block 3} \\
\hline Constant & 0.426 & 4.472 & 1.531 & 0.009 & 1 & 0.924 \\
\hline Group-CBT & -0.539 & 1.111 & 0.583 & 0.235 & 1 & 0.628 \\
\hline BDI & -0.002 & 0.090 & 0.998 & 0.001 & 1 & 0.981 \\
\hline DAS & 0.010 & 0.022 & 1.010 & 0.201 & 11 & 0.654 \\
\hline SLCS-R & -0.116 & 0.089 & 0.890 & 1.731 & 1 & 0.188 \\
\hline Categoric memories & 0.717 & 0.315 & 2.049 & 5.192 & & $0.023 * *$ \\
\hline
\end{tabular}


entered as an additional variable. This significantly increased the reliability of the overall model, $-2 \mathrm{LL}=23.34, \chi^{2}(1)=7.92, p<0.005, R^{2}=0.29$ (Cox and Snell), $R^{2}=0.40$ (Nagelkerke). Also, the Wald statistic for the number of categoric memories was significant, Wald $\chi^{2}(1)=5.19, p<0.03$. We can thus conclude that the number of categoric memories significantly predicts whether or not a patient still fulfilled criteria of major depression at Time 2. The nature of the relationship is such that more categorical memories are associated with an increased probability that the patient is still diagnosed as depressed at retest.

In a second regression analysis, the number of 'categoric memories' was replaced by the number of 'specific memories' (added in block 3). The results for this analysis are identical to those reported in Table 2 for the first three blocks (blocks 0,1 and 2). When the number of specific responses was entered in the regression in block 3 , this significantly improved the model, $-2 \mathrm{LL}=25.34, \chi^{2}(1)=6.02, p<0.02, R^{2}=0.23$ (Cox and Snell), $R^{2}=0.33$ (Nagelkerke). For all individual predictors in this last block, only the Wald statistic for the number of specific memories reached significance, Wald $\chi^{2}(1)=4.59, p<0.05$. The type of relationship is such that less specific memories are associated with an increased probability that the patient is still diagnosed as depressed at retest.

As another way to present these data, we calculated between-group contrasts for the number of categoric memories and the number of specific memories. These results show that those patients who no longer were diagnosed as suffering from major depression at Time 2 had significantly less categoric memories, $t(24)=-2.10, p<0.05$, and significantly more specific memories at baseline, $t(24)=2.02, p=0.05$, than those who were still diagnosed with depression (categoric: $M_{\text {recovered }}=1.28, M_{\text {depressed }}=3.25$; specific: $M_{\text {recovered }}=13.83, M_{\text {depressed }}=10.5$ ).

\section{Autobiographical memory and the prediction of level of depression}

Because the BDI-II was also assessed at Time 2, we were also able to test whether categoric memories are a significant predictor of the level of depression. Because the BDI-II is a continuous variable, a (hierarchical) multiple regression approach was used. Using a similar model as was used for the preceding analyses, it was found that neither categoric memories nor specific memories significantly predicted BDI-II at retest; categoric memories: $\beta=0.16, t=0.713$, n.s., specific memories: $\beta=0.13, t=0.635$, n.s. These findings replicate the results of previous studies that failed to observe predictive effects when the BDI was used as the criterion variable (Brewin et al., 1999; Dalgleish et al., 2001; see Discussion).

\section{Rumination and the prediction of diagnostic status at retest}

Previous research has shown that categoric memory (or lack of autobiographical memory specificity) is closely related to higher levels of rumination (Watkins \& Teasdale, 2001). Both can be seen as indicative of an abstract, verbally based and evaluative processing mode (Williams et al., 2007). In a prediction study by Raes et al. (2006), overgeneral memory no longer predicted the level of depression when rumination was taken into account. Additional analyses showed that rumination was a mediator of the relation between reduced AM specificity and poor outcome of depression.

To assess the predictive validity of rumination in the present study, the RSS was added to the aforementioned logistic regression in a fourth and final block. The results for this added part of the analysis are presented in Table 3b. As will be clear, the RSS had no significant effect in the regression, Wald $\chi^{2}$ $(1)=1.90, p=0.17$, whereas the effect of 'categoric memories' remained significant. Adding the RSS also did not reliably improve the regression model, $-2 \mathrm{LL}=20.77, \chi^{2}(1)=2.57, p=0.11, R^{2}=0.35$ (Cox and Snell), $R^{2}=0.50$ (Nagelkerke).

When the RSS was entered after Group-CBT (block 1) and BDI-RSS, DAS and SLCS-R (block 2), but before the number of categoric memories, rumination did improve the overall model, $2 \mathrm{LL}=26.81, \chi^{2}$ $(1)=4.44, p<0.05$, but failed to show as a significant predictor on its own, Wald $\chi^{2}(1)=3.13, p=0.08$. Also, adding categoric memories in the next block (block 4) further increased the reliability of the model, $2 \mathrm{LL}=20.77, \chi^{2}(1)=6.05, p<0.02$, and showed as a significant predictor, Wald $\chi^{2}(1)=4.02, p<0.05$. We can thus conclude that rumination was not a significant predictor of the diagnostic status of the patients, and adding this variable to the regression does not take away the predictive effect of categoric memories. Similar 
Table $3 b$

Prediction of diagnostic status by means of the RSS: regression statistics

\begin{tabular}{lccccc}
\hline Predictors & $\beta$ & S.E. $\beta$ & Exp $(\beta)$ & Wald's $\chi^{2}$ & Df \\
\hline Block 4 & & & & & \\
$\quad$ Constant & 3.406 & 5.476 & 30.138 & 0.387 & 1 \\
Group-CBT & 0.004 & 1.174 & 1.004 & 0.000 & 1 \\
BDI & 0.012 & 0.111 & 1.012 & 0.011 & 1 \\
DAS & 0.010 & 0.034 & 1.036 & 1.061 & 0.534 \\
SLCS-R & 0.035 & 0.100 & 0.920 & 0.705 & 0.916 \\
Categoric memories & -0.084 & 0.344 & 1.991 & 4.016 & 0.303 \\
RSS & -0.197 & 0.143 & 0.822 & 1.897 & 1 \\
\hline
\end{tabular}

results were observed when the 'categoric memories' variable was replaced by 'specific memories'. Finally, rumination failed to predict level of depression defined in terms of BDI-II scores at Time 2.

\section{Discussion}

The results of this study show that autobiographical memory is a reliable predictor of the course of depression. Patients who were more overgeneral had a significantly greater chance of still fulfilling criteria for depression when retested some weeks into treatment. This finding adds to previous studies in clinically depressed groups. To our knowledge, this is the first study in currently depressed patients that used the diagnostic status as the outcome variable. The predictive effect was present for both the number of specific memories and the number of categoric memories and remained when controlled for baseline measures of level of depression, self-esteem and dysfunctional attitudes. With respect to rumination, the results from our study indicated that it was not a significant predictor of diagnostic status at Time 2.

The present findings are of relevance with respect to a more recent discussion concerning the precise nature of what exactly is predicted by the AMT. As a matter of fact, depending on how recovery is defined, different patterns seem to emerge. Scott, Williams, Brittlebank, and Ferrier (1995), for example, defined recovery as a period of 8 weeks with no or minimal symptoms. Based on the patient group studied by Brittlebank et al. (1993), but now at a follow-up of 1 year, Scott et al. failed to find a predictive effect of the AMT when this alternative criterion for recovery was used. A comparison of these results with those of the original Brittlebank study, lead them to conclude that: 'Our findings highlight an important way in which outcome of a study may depend on the definition of recovery employed' (Scott et al., 1995, p. 170). Also, even within the category of symptom measures (BDI, HRSD) there exists some ambiguity about what aspects of depression are reliably predicted. In a study by Brewin et al.(1999) the findings of Brittlebank could not be replicated. An important difference, however, was that while Brittlebank employed the HRSD as the outcome measure, Brewin et al. had used the BDI. Prompted by this difference, Dalgleish et al. (2001) included the two measures and replicated both previous studies: the AMT predicted changes in the HRSD but not in the BDI. One possible explanation for the differential effects for both measures is that overgeneral memory performance is more closely related to the somatic-vegetative symptoms (as measured by the HRSD) as compared with the more cognitive symptoms (as indexed by the BDI) (Dalgleish et al., 2001, p. 338; but see Mackinger \& Svaldi, 2004). Although future research will be needed to explore the exact reasons for these differential effects depending on the nature of the outcome measure, the present findings at least reveal that when outcome is defined in terms of the diagnostic status, the AMT proves to be a reliable predictor.

One limitation of the present study is the small sample size $(n=26)$. One could argue that the absence of a predictive effect of rumination could be attributed to this lack of power. Nevertheless, related studies used similar sample sizes, including the study by Raes et al. (2006) who demonstrated the effect of rumination. Moreover, in the present study there was no significant difference on the T1 rumination scores for those who were still clinically depressed at T2 $(M=44)$ as compared with those who were not $(M=48), t=1.13$, $p=0.27$. As a matter of fact, the means were in a direction opposite to what could have been predicted. Hence, it is unlikely that the absence of a predictive affect for rumination is due to a lack of power. 
A second limitation of the present study is that the time frame for prediction was relatively short (about 24 days). The reason for choosing this time span is that experience learns that many patients drop out of the clinical range within the first weeks of therapy. The fact that clinical improvement within these first weeks of treatment can be predicted on the basis of the AMT is of clinical relevance. Nevertheless, future research should be aimed at investigating the relationship between the AMT and clinical trajectories over a series of time frames (e.g. after 4 weeks, after 3, 7 and 12 months).

\section{Acknowledgements}

We thank Luc Hamelinck, Marieke Swerts and Els Meert for their assistance in conducting this study.

\section{References}

American Psychiatric Association (1994). Diagnostic and statistical manual of mental disorders (4th ed.). Washington, DC: Author.

Beck, A. T., Steer, R. A., \& Brown, G. K. (1996). Beck Depression Inventory-second edition. San Antonio, TX: The Psychological Corporation.

Brewin, C. R., Reynolds, M., \& Tata, P. (1999). Autobiographical memory processes and the course of depression. Journal of Abnormal Psychology, 108, 511-517.

Brittlebank, A. D., Scott, J., Williams, J. M. G., \& Ferrier, I. N. (1993). Autobiographical memory in depression: State or trait marker. British Journal of Psychiatry, 162, 118-121.

Conway, M., Csank, P. A. R., Holm, S. L., \& Blake, C. K. (2000). On assessing individual differences in rumination on sadness. Journal of Personality Assessment, 75, 404-425.

Dalgleish, T., Spinks, H., Yiend, J., \& Kuyken, W. (2001). Autobiographical memory style in seasonal affective disorder and its relationship to future symptom remission. Journal of Abnormal Psychology, 110, 335-340.

First, M. B., Spitzer, R. L., Gibbon, M., \& Williams, J. B. W. (1996). Structured Clinical Interview for DSM-IV axis I disorders: Patient edition (SCID-I/P, Version 2.0). Washington, DC: American Psychiatric Press.

Hamilton, M. (1960). A rating scale for depression. Journal of Neurology, Neurosurgery and Psychiatry, $23,56-62$.

Harvey, A. G., Bryant, R. A., \& Dang, S. T. (1998). Autobiographical memory in acute stress disorder. Journal of Consulting and Clinical Psychology, 66, 500-506.

Hipwell, A., Reynolds, S., \& Pitts Crick, E. (2004). Cognitive vulnerability to post-natal depressive symptomatology. Journal of Reproductive and Infant Psychology, 22, 211-227.

Mackinger, H. F., \& Svaldi, J. J. (2004). Autobiographical memory predicts cognitive but not somatic change in sleep apnea patients vulnerable for affective disorder. Journal of Affective Disorders, 81, 17-22.

Nolen-Hoeksema, S., \& Morrow, J. (1991). A prospective study of depression and posttraumatic stress symptoms after a natural disaster: The 1989 Loma Prieta earthquake. Journal of Personality and Social Psychology, 61, 115-121.

Peeters, F., Wessel, I., Merckelbach, H., \& Boon-Vermeeren, M. (2002). Autobiographical memory specificity and the course of major depressive disorder. Comprehensive Psychiatry, 43, 344-350.

Raes, F., Hermans, D., de Decker, A., Eelen, P., \& Williams, J. M. G. (2003). Autobiographical memory specificity and affect regulation: An experimental approach. Emotion, 3, 201-206.

Raes, F., Hermans, D., \& Eelen, P. (2003). De Nederlandstalige versie van de Ruminative Response Scale (RRS-NL) en de Rumination on Sadness Scale (RSS-NL) [The Dutch version of the Ruminative Response Scale (RRS-NL) and the Rumination on Sadness Scale (RSS-NL)]. Gedragstherapie, 36, 97-104.

Raes, F., Hermans, D., Williams, J. M. G., Beyers, W., Brunfaut, E., \& Eelen, P. (2006). Reduced autobiographical memory specificity and rumination in predicting the course of depression. Journal of Abnormal Psychology, 115, 699-704.

Raes, F., Hermans, D., Van den Broeck, K., \& Eelen, P. (2005). De Nederlandstalige versie van de Dysfunctional Attitude Scale-vorm A (DAS-A-NL) [The Dutch version of the Dysfunctional Attitude Scale-A (DAS-A-NL)]. Gedragstherapie, 38, $285-293$.

Raes, F., Sienaert, P., Demyttenaere, K., Peuskens, J., Williams, J. M. G., \& Hermans, D. (in press). Over-general memory predicts stability of short-term outcome of ECT for depression. Journal of ECT.

Ramponi, C., Barnard, P. J., \& Nimmo-Smith, I. (2004). Recollection deficits in dysphoric mood: An effect of schematic models and executive mode? Memory, 12, 655-670.

Scott, J., Williams, J. M. G., Brittlebank, A., \& Ferrier, I. N. (1995). The relationship between premorbid neuroticism, cognitive dysfunction and persistence of depression: a 1-year follow-up. Journal of Affective Disorders, 33, 167-172.

Tafarodi, R. W., \& Swann, W. B., Jr. (2001). Two-dimensional self-esteem: Theory and measurement. Personality and Individual Differences, 31, 653-673.

Van der Does, A. J. W. (2002). Handleiding bij de Nederlandse bewerking van de BDI-II [Manual of the Dutch version of the BDI-II]. San Antonio, TX/Lisse, The Netherlands: The Psychological Corporation/Swets Test Publishers.

Vandromme, H., Hermans, D., Spruyt, A., \& Eelen, P. (2007). Dutch translation of the Self-Liking/Self-Competence Scale-Revised: A confirmatory factor analysis of the two-factor structure. Personality and Individual Differences, 42, $157-167$. 
Van Groenestijn, M. A. C., Akkerhuis, G. W., Kupka, R. W., Schneider, N., \& Nolen, W. A. (1999). Gestructureerd Klinisch Interview voor de vaststelling van DSM-IV As I Stoornissen [Structured Clinical Interview for DSM-IV axis I disorders]. Lisse, The Netherlands: Swets \& Zeitlinger.

Watkins, E., \& Teasdale, J. D. (2001). Rumination and overgeneral memory in depression: Effects of self-focus and analytic thinking. Journal of Abnormal Psychology, 110, 353-357.

Weissman, A. N., \& Beck, A. T. (1978). Development and validation of the Dysfunctional Attitude Scale: A preliminary investigation. Paper presented at the meeting of the association for the advancement of behavior therapy, Chicago.

Williams, J. M. G., Barnhofer, T., Crane, C., Hermans, D., Raes, F., Watkins, E., et al. (2007). Autobiographical memory specificity and emotional disorder. Psychological Bulletin, 133, 122-148.

Williams, J. M. G., \& Broadbent, K. (1986). Autobiographical memory in suicide attempters. Journal of Abnormal Psychology, 95, 144-149. 